\title{
Parallel-Beam CT Reconstruction Based on Mojette Transform and Compressed Sensing
}

\author{
Wen Hou and Cishen Zhang
}

\begin{abstract}
A novel frame of parallel-beam Computerized Tomography (CT) reconstruction is proposed. The CT projections are firstly converted to Mojette projections. Then 1D Fourier transform is applied to the modified Mojette projections, followed by an exact mapping of the gained Fourier coefficients to the 2D Fourier domain of the scanning object. Finally the scanning object can be reconstructed from the partial Fourier coefficients by compressed sensing (CS). Experimental results show that using this frame, the purpose of reducing the radiation dosage during $\mathrm{CT}$ examinations without compromising the image quality can be achieved.
\end{abstract}

Index Terms-Computerized tomography, parallel-beam, mojette transform, compressed sensing.

\section{INTRODUCTION}

Computerized Tomography (CT) is a powerful medical tool which is helpful for the diagnosis of doctors and thus can benefit patients a lot. In general, the image quality of CT is proportional to the radiation dosages. But increased radiation dosages raise the risk of cancer, which becomes an obstacle for CT development. Hence, it is meaningful and challenging work to reduce the radiation dosage during $\mathrm{CT}$ examinations without compromising the image quality.

Classical tomography is concerned with the recovery of the scanning object from a set of projections, which is the Radon transform (RT) [1] of the object. As Radon transform is continuous both for the object under scan and the projections themselves, it has to be sampled to adjust to practical application, resulting in ill-posedness. Mojette transform [2] is a both experimentally and computationally viable discretization of Radon transform. It regularizes the ill-posedness of inverse Radon transform and allows for an exact reconstruction in the discrete domain with a finite number of projections. Despite its advantages, Mojette transform has two main shortcomings. One is that it is not compatible with the physical acquisition of $\mathrm{CT}$, and this problem is addressed in paper [3], in which the authors design a linear system to calculate the Mojette projections from a Radon acquisition. The other is that the angle and bin set is different from practical CT machine, i.e., classical tomograph acquires are equally distributed over $2 \pi$ with a fixed number of bins onto each projection, while Mojette transform is defined over Farey angles with varying orientation and number of bins onto each projection. A lot of research ${ }^{[4]}$ has been done, committed to applying Mojette

Manuscript received October 20, 2012; revised November 24, 2012.

Wen Hou and Cishen Zhang are with the Faculty of Engineering and Industrial Sciences, Swinburne University of Technology, Vic 3122, Australia (e-mail: wenhou@swin.edu.au; cishenzhang@swin.edu.au) transform to classical tomographic data, which implies the advantage of Mojette transform on one hand and improves the potential of Mojette transform in practical application on the other hand. Paper [4] claims that any set of real, acquired tomographic data can be rebinned into a compatible Mojette projection space, without any loss of reconstruction power.

After getting the projections, the next step is the reconstruction problem. M. Katz [5] presents some reconstructubility theorems for discrete images and projections. A lot of work has also been done to recover object based on Mojette projections, committed to the improvement of the accuracy. Since we try to reduce the radiation dosage as well, a newly developed technique called compressed sensing (CS) is used. CS attempts to reconstruct signals from significantly fewer samples than were traditionally thought necessary (e.g., Nyquist sampling theorem) [6]. CT imagery happens to meet the two key requirements for successful application of CS: it is compressible in some transform domain and the scanner gets encoded samples. Applying CS to CT reconstruction, the high-accuracy reconstruction with low-dosage radiation is expected to be obtained [7].

The main contribution of the paper is that a novel reconstruction frame, i.e. the combination of Mojette transform and CS, is proposed in the interest of accuracy and radiation. A detailed description and explanation about how to modify the Mojette projections for the application of CS is given. The rest of paper is organized as follows. In Section II, the Mojette transform and CS is briefly introduced, and the relation between Mojette transform and the 2D Fourier coefficients of the scanning object is formulated, at last the detailed reconstruction algorithm is described. Section III gives the experimental results to validate the accuracy, low-dosage radiation and noise tolerance of the proposed frame. Conclusions are drawn in Section IV.

\section{RECONSTRUCTION FRAME BASED ON MOJETTE TRANSFORM AND COMPRESSED SENSING}

\section{A. Mojette Transform}

For simplicity, we assume the scanning object to be $f(x, y)$ with the size $N \times N$ ( $N$ is even). CT scanner maps the $2 \mathrm{D}$ object into a set of $1 \mathrm{D}$ projection lines, which forms the sinogram. Each line is the Radon transform of $f$ for a given angle $\theta$ and a module $\rho$ [1], defined by:

$$
R_{\theta}(\rho)=\int_{-\infty}^{+\infty} \int_{-\infty}^{+\infty} f(x, y) \delta(\rho-x \cos \theta-y \sin \theta) d x d y
$$


where $\theta$ and $\rho$ are respectively the angular and radial coordinates of the projection line $(\theta, \rho)$, and $\delta$ is the Dirac's delta function.

The Dirac-Mojette transform is an exact discretization of the Radon transform. It is defined over angles $=$ $\tan ^{-1}(q / p)$, where $p$ and $q$ are relatively prime integers. As the Farey series of order $K$, denoted by $F_{k}$, is the set of all fractions in lowest terms between 0 and $\infty$, whose denominators don't exceed $K$, e.g. $F_{4}=\left[\frac{0}{1}, \frac{1}{4}, \frac{1}{3}, \frac{1}{2}, \frac{2}{3}, \frac{3}{4}, \frac{1}{1}, \frac{4}{3}, \frac{3}{2}, \frac{2}{1}, \frac{3}{1}, \frac{4}{1}, \frac{1}{0}\right]$, we can use it to give a set of discrete angles between $\left[0, \frac{\pi}{2}\right]$ and obtain the angles over $[0, \pi]$ by symmetry. The Dirac-Mojette transform is defined as:

$$
M_{p, q}(\rho)=\sum_{x} \sum_{y} f(x, y) \Delta(\rho-p x-q y)
$$

The geometry of Dirac-Mojette projector is shown in Fig.1(a), where the pixel is summed to its corresponding bin if and only if the X-ray passes through the centre of the pixel[8].

Fourier Slice Theorem claims that the 1D Fourier transform of the projections is equal to the $2 \mathrm{D}$ Fourier transform of the image evaluated on the line that the projection was taken on. In this paper, it is formulated that the 2D Fourier values of the scanning object can be obtained exactly through Mojette transform. Let $(u, v)$ be the coordinate in the frequency domain, then the 2D discrete Fourier transform of $f(x, y)$ is:

$$
F(u, v)=\sum_{x} \sum_{y} f(x, y) e^{-j \frac{2 \pi}{N}(u x+v y)}
$$

The 1D discrete Fourier transform of $M_{\mathrm{p}, \mathrm{q}}(\rho)$ is:

$$
F M_{p, q}(r)=\sum_{\rho} M_{p, q}(\rho) e^{-j \frac{2 \pi}{L} \rho r}
$$

where $L$ is the projection size, and $L=(N-1)(|p|+$ $q+1$. Combining (2) and (4), we can get:

$$
F M_{p, q}(r)=\sum_{x} \sum_{y} f(x, y) e^{-j \frac{2 \pi}{L} r(p x+q y)}
$$

Comparing (3) and (5), it is hard to map $F M_{p, q}(r)$ to $F(u, v)$ directly. Considering the periodicity of Fourier transform, we can merge the items gained by (2) with the same $\bmod (p x+q y, N)$, then projection size can be reduced to $N$. So

Its 1D Fourier transform is:

$$
M_{p, q}^{\prime}(\rho)=\left.\sum_{x} \sum_{y} f(x, y)\right|_{\rho=\bmod (p x+q y, N)}
$$

$$
\begin{aligned}
F M_{p, q}^{\prime} & =\sum_{x} \sum_{y} f(x, y) e^{-j \frac{2 \pi}{N} r[\bmod (p x+q y, N)]} \\
& =\sum_{x} \sum_{y} f(x, y) e^{-j \frac{2 \pi}{N}[\bmod (r p x+r q y, N)]}
\end{aligned}
$$

The mapping relationship is revealed over the analysis of (3) and (7):

$$
\forall x, y, \bmod (u x+v y-r p x-r q y, N)=0
$$

Hence, the 1D Fourier transform of the modified Mojette projections taken over angles $\theta=\tan ^{-1}(q / p)$ can be mapped to the $2 \mathrm{D}$ Fourier plane of the scanning object with the mapping relationship:

$$
\bmod (u-r p)=0 \quad \& \quad \bmod (v-r q, N)=0
$$

As a result, we can get the partial Fourier coefficients where $\bmod (q u-p v, N)=0$. The Fourier coefficients gained through the mapping of the Mojette projections in Fig. 1. (a) $(N=6, \tan \theta=1 / 2)$ are marked with star in Fig. 1(b).
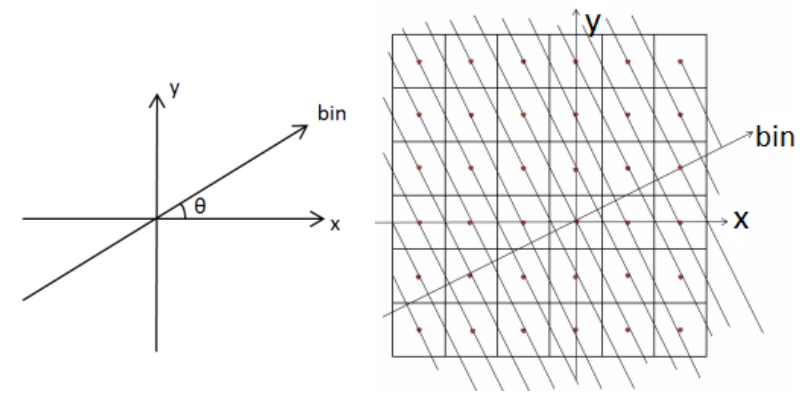

(a) Dirac-Mojette Projection of a $6 \times 6$ image

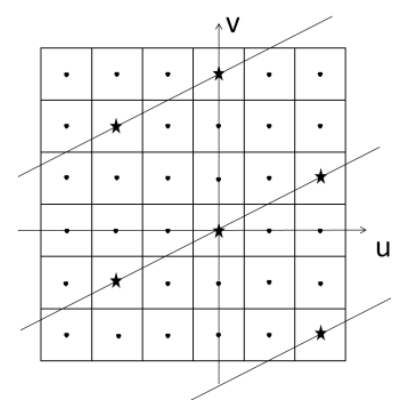

(b) The corresponding Fourier values of the Mojette projections

Fig. 1. The geometry of Mojette transform and the mapping relationship in Fourier domain

\section{B. Compressed Sensing}

Compressed sensing is a recently developed theory of signal recovery from highly incomplete information, and thus will be employed here to reconstruct the image from the partial Fourier coefficients. The central idea of CS is that a sparse or compressible signal $x \in R^{N}$ can be recovered from a small number of linear measurements $b=A x \in$ $R^{K}, K \ll N$. The reconstruction can be achieved by solving the well-known basis pursuit problem [9]-[10]:

$$
\min \|x\|_{1} \text { s.t. } A x=b
$$

With the noisy and incomplete samples, an appropriate relaxation is given by:

$$
\min \|x\|_{1} \quad \text { s.t. }\|b-A x\|_{2} \leq \sigma
$$

where $\sigma>0$ is related to the noise.

To gain more effective reconstruction results, a simple and fast algorithm called RecPF (Reconstruction from Partial Fourier data) [11] is adopted. It uses an alternating minimization scheme to solve the following model:

$$
\min _{I} \quad T V(I)+\lambda\|\psi I\|_{1}+\mu\left\|F_{p}(I)-f_{p}\right\|^{2}
$$

where $I$ is the image to be reconstructed, $T V(I)$ is the total variation regularization term, $\psi$ is a sparsifying basis (e.g., wavelet basis), $F_{p}$ is a partial Fourier matrix and $f_{p}$ denotes the partial Fourier coefficients. As the main computation of solving the model only involves shrinkage and fast Fourier transforms, the reconstruction process is quite fast. 


\section{Proposed Frame}

The proposed frame contains two stages. At the first stage, the sinogram is converted to Mojette projections using the linear system designed in paper [3], then their Fourier

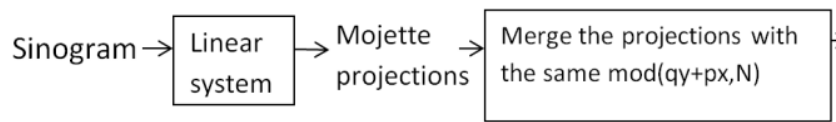

values are mapped to the 2D Fourier domain of the object. At the second stage, CS is applied to the partial Fourier coefficients for the recovery of the object. The flowchart is shown as below.

Fig. 2. Flowchart of the proposed method

Based on the above analysis, it is known that the exact Fourier coefficients can be obtained through Mojette transform, which is very beneficial for the reconstruction accuracy. The application of CS afterwards reduces the Fourier samples needed for a perfect recovery. Hence, good reconstruction results can be expected using the proposed frame when the CT scanning just covers limited number of view angles.

\section{EXPERIMENTAL RESULTS AND DISCUSSION}

\section{A. Noise-free reconstruction}

In this part, two groups of experiments are designed over the same Farey angle sets and different Farey angle sets, respectively. The performance of different methods is measured through the quantities listed below: the error in the reconstructed image relative to the original image (Err), mean square error (MSE) and signal-to-noise ratio (SNR). Let $I_{0}$ denote the original image, $I$ the reconstructed image, then the definitions of the two quantities are:

$$
\begin{gathered}
E r r=\sqrt{\frac{\sum_{i} \sum_{j}\left[I(i, j)-I_{0}(i, j)\right]}{\sum_{i} \sum_{j} I_{0}(i, j)^{2}}} \\
M S E=\operatorname{mean}\left[\left(I(i, j)-I_{0}(i, j)\right)^{2}\right] \\
S N R=10 \times \lg \frac{\sum_{i} \sum_{j}(I(i, j)-\operatorname{mean}(I))^{2}}{\sum_{i} \sum_{j}\left(I(i, j)-I_{0}(i, j)\right)^{2}}
\end{gathered}
$$

Firstly, the experiments are conducted over the same Farey angle sets. We present simulation results of the $256 \times 256$ phantom image reconstrucion obtained by MCS and two other methods: FBP and Mift, where FBP uses filtered backprojection algorithm over the Radon projections and Mift applies direct inverse Fourier transform to the Mojette projections. The projections are taken over the Farey angles. Fig. 3 shows the reconstruction results of the three methods for $F_{8}$ projetions, from which we can see that FBP result has disturbing artifacts due to limited projections, and Mift can't recover the image from insufficient Fourier samples. It is obvious that MCS does the almost exact construction. The Err of the three different methods as a function of the order of Farey series is plot in Fig.4. We can see that MCS has already got the good reconstruction result with $F_{4}$ projections, where the Err of Mift and FBP is still larger than 50\%. Fig.4 also shows that FBP can gain better results than Mift with the increasing number of projections.
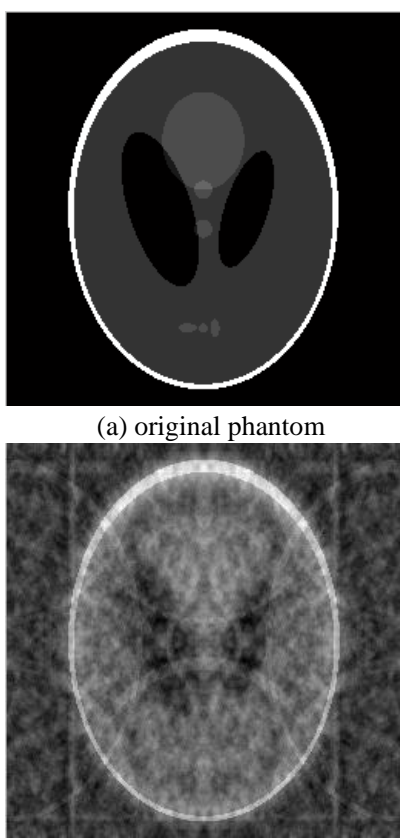

(c) Mift (a) original phantom

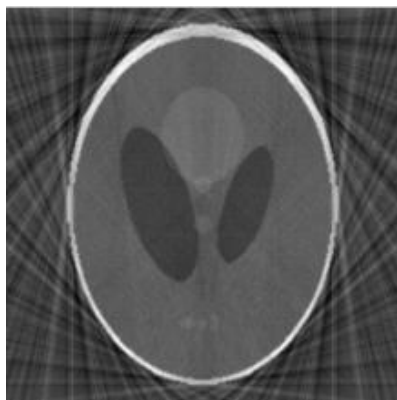

(b) FBP

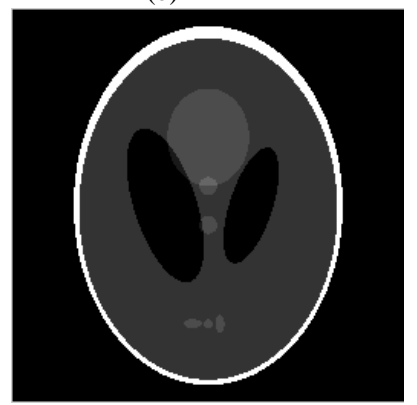

(d) MCS
Fig. 3. Reconstruction results of the three methods from the projections taken over $\mathrm{F}_{8}$

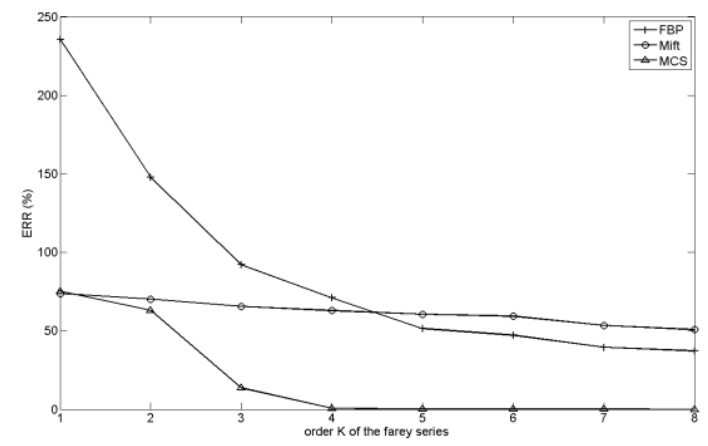

Fig. 4. A comparison of Err gained by different methods with the increasing order of the Farey series

Then the experiments are conducted over different Farey angle sets. The Mojette filtered backprojection algorithm (MFBP) [12] is used here for comparison. We use the same experimental image as MFBP, a $128 \times 128$ phantom image consisting of a $17 \times 17$ square object with unitary value whereas boundaries are only half valued. Table I lists the MSE of MCS and MFBP reconstruction results with different orders of Farey series. We can see that MCS can gain better results over $F_{1}$ angle set with just 4 projection lines than MFBP over $F_{32}$ angle set with 1296 projection lines. Here are the reconstruction results in Fig.5. It is shown that the result of MFBP over $F_{32}$ angle in (b) has some disturbing artefacts, while MCS gives clear and exact 
reconstruction result with only 4 projection lines. Fig.5 (d) shows the line mask of the partial Fourier coefficients which are the input of CS. If the X-ray tube current is fixed, the total radiation exposure of the target is proportional to the number of view angles. Hence, the conclusion is drawn that less projections are needed for the reconstruction with the help of CS, which means shorter scanning time and lower radiation dosage, and thus will benefit the patients more.

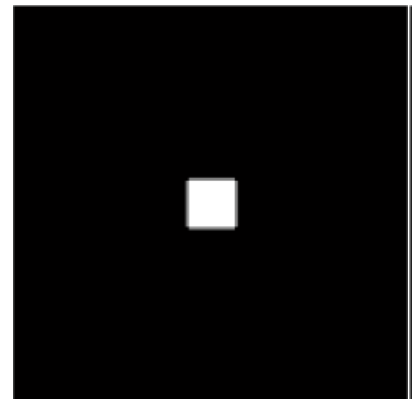

(a) original phantom

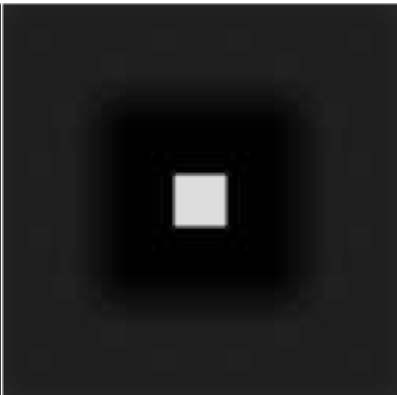

(b) MFBP over $F_{32}$

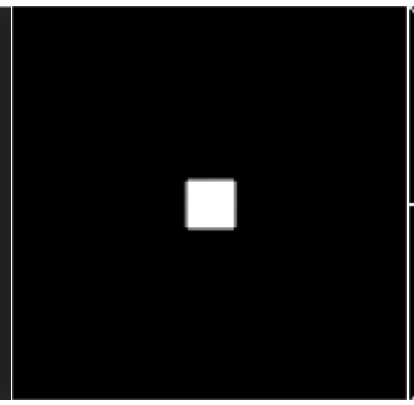

(c) MCS over $\mathrm{F}_{1}$

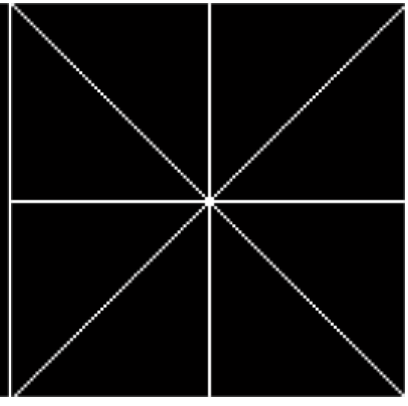

(d) line mask

Fig. 5. Reconstruction results of MCS and MFBP

TABLE I: MSE OF MCS AND MFBP RECONSTRUCTION RESULTS

\begin{tabular}{c|cccc|ccc}
\hline \hline Method & \multicolumn{4}{|c|}{ MCS } & \multicolumn{3}{c}{ MFBP } \\
\hline F & $F_{1}$ & $F_{2}$ & $F_{3}$ & $F_{4}$ & $F_{32}$ & $F_{64}$ & $F_{128}$ \\
\#proj & 4 & 8 & 16 & 24 & 1296 & 5040 & 20088 \\
MSE & $1.69 \times 10^{-7}$ & $6.95 \times 10^{-8}$ & $7.88 \times 10^{-9}$ & $1.23 \times 10^{-10}$ & 0.01322 & $2 \times 10^{-5}$ & 0 \\
\hline \hline
\end{tabular}

\section{B. Noise tolerance}

This experiment is designed to test the noise tolerance of the proposed frame. We generate our test sets using the $256 \times 256$ Shepp-Logan phantom image and FBP is employed here for comparison. The Gaussian noise is added to the Mojette projections in MCS and the Radon projections in FBP, respectively. In both methods, the projections are taken over $\mathrm{F}_{8}$ angle set, i.e., 88 projections. As the noise is produced randomly, the quantitative assessment (Err and SNR) is the mean value of the 20 groups of experimental data, and the results are shown in Table II. We can see that for the Gaussian noise $(0,0.001)$, i.e. with mean 0 and standard deviation 0.001 , MCS can gain a much better reconstruction than FBP, the results of which are shown in Fig.6. But for $(0,0.01)$ noise, the result of FBP barely changes while the reconstruction quality of MCS reduces a lot, which means FBP is robust to noise and MCS is sensitive. The reason is that MCS is based on noisesensitive Fourier transform. When $(0,0.01)$ noise is added to the projections, the noise of its Fourier coefficients becomes $(0,0.1)$ for a $256 \times 256$ image, verified in Fig.7. Since CS has certain ability to suppress noise, we get the conclusion that though sensitive, the proposed frame can deal with small noise effectively. It will be the future work to deal with the noisier projections [13].

\begin{tabular}{ccccc}
\multicolumn{3}{c}{ TABLE II: NOISE RESPONSE OF FBP AND MCS } \\
\hline \hline \multirow{3}{*}{ Noise } & \multicolumn{3}{c}{ FBP } & \multicolumn{2}{c}{ MCS } \\
& Err & SNR & Err & SNR \\
\hline$(0,0.001)$ & $37.1577 \%$ & 7.3586 & $2.1929 \%$ & 31.9393 \\
$(0,0.01)$ & $37.1587 \%$ & 7.3584 & $23.1656 \%$ & 11.4631 \\
\hline \hline
\end{tabular}

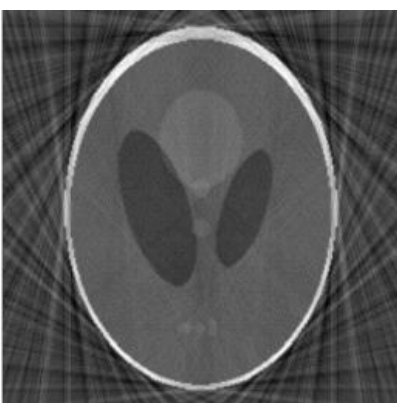

(a) FBP

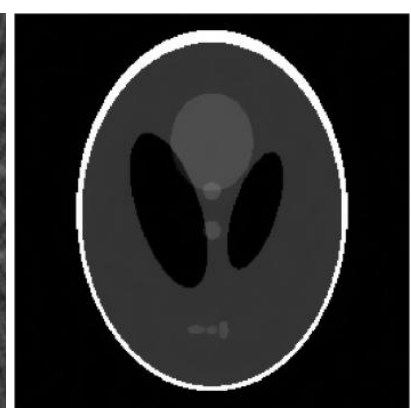

(b) MCS
Fig. 6. Reconstruction results from the $\mathrm{F}_{8}$ projections with Gaussian noise $(0,0.001)$

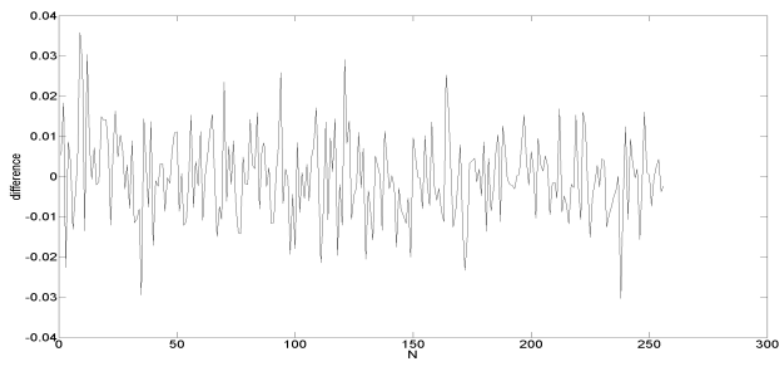

(a) the noise of projections

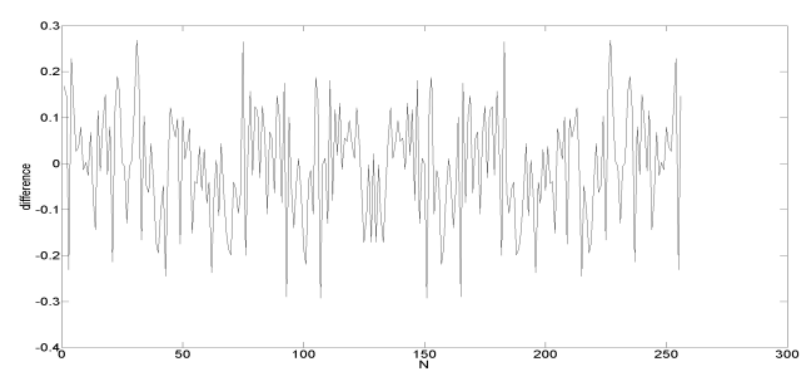

(b) the corresponding noise of the Fourier values

Fig. 7. Noise of the projections and the Fourier values 


\section{CONCLUSION}

In this paper, a novel frame for parallel-beam CT reconstruction is presented. Firstly, the sinogram is converted to the projections gained through Mojette transform, an exact discretization of Radon transform. On each view angle, the projections are summed up under some principles. Then the 1D Fourier coefficients of the merged projections are mapped to the 2D Fourier domain of the object. Finally compressed sensing is employed to deal with the partial Fourier coefficients and can recover the object very well and suppress the small noise effectively. Experimental results have demonstrated the advantages of the proposed method. With the presence of Mojette transform and compressed sensing, the purpose of reducing the radiation dosage during $\mathrm{CT}$ examinations without compromising the image quality is achieved.

\section{REFERENCES}

[1] P. Toft, "The Radon Transform: Theory and Implementation," Ph.D thesis, Department of Mathematical Modelling, Section for Digital Signal Processing, Technical University of Denmark, 1996.

[2] J. P. Guedon, D. Barba, and N. Burger, "Psychovisual image coding via an exact discrete Radon transform," in Proc. of the SPIE - The International Society for Optical Engineering, 1995, vol. 2501, pp. 562-572.

[3] B. Recur, P. Desbarats, and J. P. Domenger, "Radon and Mojette projections' equivalence for tomographic reconstruction using linear systems," in WSCG 2008 Communication Papers, 2008, pp. 191-198.

[4] H. Fayad, J. P. Guedon, I. Svalbe, Y. Bizais, and N. Normand, "Applying Mojette discrete Radon transforms to classical tomographic data," in Proc. of SPIE, 2008, vol. 6913, pp. 1605-7422.

[5] M. Katz, "Questions of Uniqueness and Resolution in Reconstruction from projections," Lect. Notes Biomath, vol. 26, Springer-Verlag, Berlin, Heidelberg, New York, 1979.

[6] M. Lustig, D. L. Donoho, J. M. Santos, and J. M. Pauly, "Compressed sensing MRI," IEEE Signal Processing Magazine, pp. 72-82, 2008.

[7] X. Han, J. G. Bian, and D. R. Eaker, "Algorithm-Enabled Low-Dose Micro-CT Imaging," IEEE Trans. Medical Imaging, vol. 30, no. 3, pp. 606-620, 2011.

[8] S. Chandra, N. Normanda, A. Kingston, J. Guedon, and I. Svalbe. (2010). Fast Mojette transform for discrete tomography. Elsevie Signal Processing Submitted June (in Review). [Online]. Available: http://arxiv.org/abs/1006.1965v1
[9] E. Candes, J. Romberg, and T. Tao, "Robust uncertainty principles: Exact signal reconstruction from highly incomplete frequency information," IEEE Trans. Inform. Theory, vol. 52, no. 2, pp. 489509, 2006.

[10] D. L. Donoho, Y. Tsaig, I. Drori, and J. L. Starck, "Sparse solution of underdetermined systems of linear equations by stagewise orthogonal matching pursuit," IEEE Trans. Inform. Theory, vol. 58, no. 2, pp. 1094-1121, 2012.

[11] J. F. Yang, Y. Zhang, and W. Yin. A fast TVL1-L2 minimization algorithm for signal reconstruction from partial Fourier data. IEEE Journal of Selected Topics in Signal Processing Special Issue on compressed Sensing. [Online]. Available: http://www.caam.rice.edu/ optimization/L1/RecPF/

[12] M. Servieres, N. Normand, J. P. Guedon, and Y. Bizais, "The Mojette transform: Discrete angles for tomography," Electronic Notes in Discrete Mathematics, vol. 20, pp. 587- 606, 2005.

[13] B. Recur, P. Desbarats, and J. P. Domenger, "Mojette reconstruction from noisy projections," in Proc. 2010 2nd International Conference on Image Processing Theory Tools and Applications (IPTA), 2010, pp. 201-206.

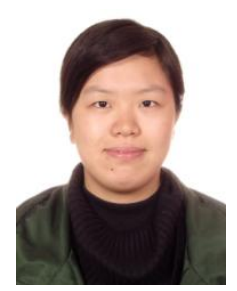

Wen Hou received the B.Eng. degree from Nanjing University of Aeronautics and Astronautics, China. She is currently doing Ph.D. in the Faculty of Engineering and Industrial Sciences, Swinburne University of Technology, Australia. Her research interests include medical imaging and reconstruction.

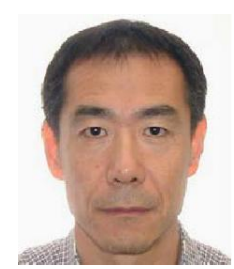

Cishen Zhang received the B.Eng. degree from Tsinghua University, China, in 1982 and Ph.D. degree in Electrical Engineering from Newcastle University, Australia, in 1990. Between 1971 and 1978, he was an Electrician with Changxindian (February Seven) Locomotive Manufactory, Beijing, China. He carried out research work on control systems at Delft University of Technology, The Netherlands, from 1983 to 1985 . After his Ph.D. study from 1986 to 1989 at Newcastle University, he was with the Department of Electrical and Electronic Engineering at the University of Melbourne, Australia as a Lecturer, Senior Lecturer and Associate Professor and Reader till October 2002. He is currently with the Faculty of Engineering and Industrial Sciences, Swinburne University of Technology, Australia. His research interests include signal processing, medical imaging and control. 